\title{
Fatores de risco cardiovascular em moradores de uma região atendida por uma Unidade Básica de Saúde
}

\author{
Cardiovascular risk factors in residents of a region served by a Basic Health Unit
}
Pollyana de Rezende Castilho ${ }^{* 1}$, Camilo Amaro de Carvalho ${ }^{1}$, Flávia Euzébio Domingues ${ }^{1}$, Letícia Peixoto Lessa ${ }^{1}$, Mariana Oliveira Ferreira ${ }^{1}$, Marina de Oliveira Ramalho Tarbes ${ }^{1}$, José Reinaldo Oliveira Junior ${ }^{1}$ e Luciana Moreira Lima ${ }^{1}$

${ }^{1}$ Departamento de Medicina e Enfermagem, Universidade Federal de Viçosa (UFV), Viçosa, Brasil

\begin{abstract}
Resumo
As doenças cardiovasculares (DCV) são a principal causa de óbitos em todas as faixas etárias no mundo. Pode-se afirmar que os gastos públicos para o tratamento dessas doenças são elevados. Desse modo, o desenvolvimento de estratégias preventivas torna-se importante não só para o benefício do paciente, mas também do ponto de vista financeiro. Este estudo analisou a prevalência de tabagismo, obesidade, aumento da circunferência da cintura, hipertensão arterial sistêmica (HAS), diabetes, sedentarismo e história familiar para DCV, além dos hábitos alimentares de residentes do bairro Barrinha, em Viçosal MG. Dentre os 121 participantes do estudo foi observada alta prevalência de história familiar para DCV e sedentarismo. A prevalência de tabagismo, obesidade e HAS foi significativamente maior em adultos comparada à verificada em crianças e adolescentes. A média do IMC obtida para os adultos permite classificá-los em pré-obesos e a circunferência de cintura foi considerada aumentada. Diante da alta prevalência de FRC modificáveis na população estudada, faz-se necessário o planejamento e concretização de ações de cuidado integral com enfoque na prevenção das DCV junto à comunidade estudada.
\end{abstract}

Palavras-chave: Doenças cardiovasculares, fatores de risco, prevenção.

\begin{abstract}
Cardiovascular diseases (CVD) are the leading cause of death in all age groups and both sexes in worldwide. It can be argued that public spending for the treatment of these diseases are high. Thus, the development of preventive strategies becomes important for the benefit of the patient and also from a financial standpoint. This study examined the prevalence of smoking, obesity, increased waist circumference, high blood pressure (hypertension), diabetes, sedentary lifestyle and family history of CVD, beyond the eating habits of residents of Barry neighborhood in Viçosa/MG. Among the 121 participants studied high prevalence of family history of CVD and physical inactivity was observed. The prevalence of smoking, obesity and hypertension was significantly higher in adults compared to those in children and adolescents. The average IMC of adults can be classified as pre-obese and waist circumference was considered increased. Given the high prevalence of modifiable CRF in this population, it is necessary planning and implementation of actions integrated care focusing on prevention of CVD in the community studied.
\end{abstract}

Keywords: Cardiovascular diseases, risk factors, prevention.

* pollyrc@yahoo.com.br

Recebido: 20/03/2014 Aceito: 20/03/2014 


\section{Introdução}

A s doenças cardiovasculares (DCV) são a principal causa de óbitos em todas as faixas etárias, tanto do sexo feminino quanto do sexo masculino no Brasil $^{1}$ e no mundo ${ }^{2}$, sendo o maior número de mortes atribuídos à doença arterial coronariana (DAC) e ao acidente vascular encefálico $(\mathrm{AVE})^{2}$. Estudos epidemiológicos apontam aumento da mortalidade causada por DCV em países em desenvolvimento ${ }^{3}$. No Brasil, entre as décadas de 1980 e 1990, houve um aumento de 13\% do coeficiente de mortalidade por $\mathrm{DCV}^{3}$. Não há projeções futuras de melhora, devido principalmente ao aumento da longevidade, mudanças inadequadas no estilo de vida e condições socioeconômicas insatisfatórias ${ }^{3}$.

Os gastos públicos para o tratamento dessas doenças são elevados ${ }^{4}$, dentre as despesas destacam-se a necessidade de internações hospitalares, incapacitação, procedimentos diagnósticos e terapêuticos, acompanhamento médico e farmacológico continuado ${ }^{4}$. Desse modo, o desenvolvimento de estratégias preventivas torna-se importante não só para o benefício do paciente, mas também do ponto de vista financeiro ${ }^{3,4}$. Porém, prevenção é um grande desafio devido à etiologia multifatorial das $\mathrm{DCV}$, atribuídas principalmente à associação com fatores de risco cardiovascular (FRC) ${ }^{3}$, os quais são modificáveis ou não modificáveis, tais como idade e hereditariedade. O estudo INTERHEART ${ }^{5}$, apontou três fatores de proteção cardiovascular (exercício físico, ingestão de bebidas alcoólicas em pequena quantidade e consumo diário de vegetais e frutas) e seis FRC modificáveis (HAS, DM, obesidade, tabagismo, estresse psicológico e dislipidemia, sendo a última responsável por mais de dois terços do risco de infarto ${ }^{5}$ ) responsáveis por mais de $90 \%$ do risco atribuível a $\mathrm{DCV}^{5}$. Estudos evidenciaram que quanto maior a associação de FRC em um indivíduo, maior a probabilidade do desenvolvimento da doença e que a modificação desses fatores pode alterar o índice de sua progressão com redução da mortalidade ${ }^{6}$. Portanto, é de extrema importância identificar os FRC e controlá-los de modo eficaz.

Este estudo analisou a prevalência de tabagismo, obesidade, aumento da circunferência da cintura, hipertensão arterial sistêmica (HAS), diabetes, sedentarismo e história familiar para $\mathrm{DCV}$, além dos hábitos alimentares de residentes em um bairro atendido por uma Unidade Básica de Saúde (UBS) no município de Viçosa/MG. Objetivou-se estratificar os principais FRC da região, assim como desenvolver intervenções para o controle dos principais fatores predisponentes, além do aprimoramento da prática de educação em saúde.

\section{Material e métodos}

O projeto foi submetido ao Comitê de Ética em Pesquisa com Seres Humanos (CEPH) da UFV. Aos indivíduos selecionados para participar do estudo, esclareceu-se os objetivos do projeto e, àqueles concordantes, requereu-se a assinatura do Termo de Consentimento Livre e Esclarecido (TCLE) aprovado pelo CEPH. Uma ficha clínica contendo dados importantes para a análise dos resultados foi preenchida em todos os casos.

Para responder aos objetivos propostos, realizou-se um cálculo de amostra com os indivíduos residentes na cidade de Viçosa/MG, fundamentado em princípios epidemiológicos e estatísticos, estabelecendo-se o número mínimo de 96 indivíduos. Participaram do estudo 121 indivíduos, adultos e crianças, estratificados de acordo com o sexo e a idade, para análise dos FRC.

Foi definido como grupo de estudo os residentes do bairro Barrinha de Viçosa/MG assistidos pela Unidade de Atendimento Primário (UAP) São José e estendeu-se a pesquisa a moradores do bairro Cidade Nova assistidos pela mesma UAP. Residências foram selecionadas aleatoriamente ou por sugestão de Agentes Comunitários de Saúde (ACS) com o critério de maior quantidade de moradores dentro da faixa etária determinada.

Foram incluídos no estudo indivíduos de ambos os sexos, na faixa de idade de 5 a 99 anos, que aceitaram participar do estudo voluntariamente. Aos indivíduos menores de 18 anos foi solicitada uma autorização formal do responsável legal. Foram excluídos indivíduos portadores de doenças intercorrentes como distúrbios da coagulação, doenças renais, hepáticas, autoimunes, câncer e insuficiência cardíaca.

O preenchimento da ficha foi realizado com auxílio de balança digital para o peso, esfigmomanômetro aneroide e estetoscópio para realização de duas aferições da pressão arterial (PA) em braço direito e obtenção da média aritmética, além de fita métrica para obtenção da circunferência abdominal (CA) e altura. Em caso de respostas pouco confiáveis - em entrevistas realizadas com crianças, idosos ou portadores de demências - para questionamentos sobre hábitos alimentares, uso de medicamentos e história familiar, apoiou-se em dados fornecidos por familiares.

Para a análise estatística foram realizados: os testes t-Student para variáveis com distribuição normal; e Mann-Whitney para variáveis não paramétricas. Na avaliação da correlação, utilizou-se o teste de correlação de Pearson. O nível de significância adotado para o estudo foi de 5\%. Os programas Sigma Stat versão 1.0 e Excell versão 3.0 foram utilizados para realizar as análises e plotar os gráficos, respectivamente.

\section{Resultados}

Dos 121 participantes do estudo 86 (71,1\%) eram adultos e $35(28,9 \%)$ crianças e adolescentes. Foi observada uma alta prevalência de história familiar para doenças cardiovasculares e sedentarismo entre os indivíduos estudados. A tabela 1 e a figura 1 apresentam a preva- 


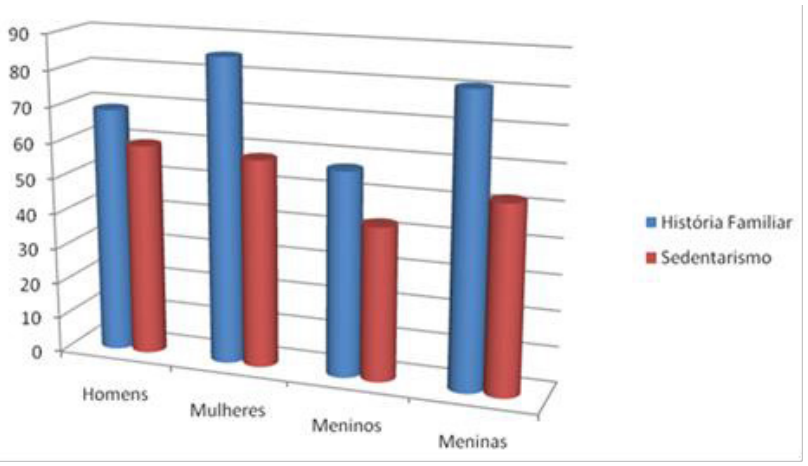

Figura 1. Fatores de risco mais prevalentes na população estudada.

Tabela 1. Fatores de risco cardiovascular nos participantes do estudo

\begin{tabular}{|c|c|c|c|c|}
\hline Parâmetro & Total & Adultos & $\begin{array}{c}\text { Crianças e } \\
\text { adolescentes }\end{array}$ & p \\
\hline $\mathrm{n}$ & $\begin{array}{c}121 \\
(100 \%)\end{array}$ & $\begin{array}{c}86 \\
(71,1 \%)\end{array}$ & $\begin{array}{c}35 \\
(28,9 \%)\end{array}$ & - \\
\hline Idade (anos) & $\begin{array}{c}31 \\
(13-56,5)\end{array}$ & $\begin{array}{c}41 \\
(26,8-63,3)\end{array}$ & $\begin{array}{c}10 \\
(7-11,8)\end{array}$ & $<0,001^{\text {(a) }}$ \\
\hline $\operatorname{IMC}\left(\mathrm{Kg} / \mathrm{m}^{2}\right)$ & $\begin{array}{c}22,3 \\
(18,5-27,9)\end{array}$ & $\begin{array}{c}25,7 \\
(21,9-28,6)\end{array}$ & $\begin{array}{c}16,9 \\
(15,2-18,1)\end{array}$ & $<0,001^{\text {(a) }}$ \\
\hline Circunferência da cintura $(\mathrm{cm})$ & $81,9 \pm 18,5$ & $89,9 \pm 13,8$ & $62,2 \pm 12,5$ & $<0,001^{\text {(b) }}$ \\
\hline Tabagismo - n (\%) & $\begin{array}{c}16 \\
(13,2 \%)\end{array}$ & $\begin{array}{c}15 \\
(17,4 \%)\end{array}$ & $\begin{array}{c}1 \\
(2,9 \%)\end{array}$ & $0,037^{(c)}$ \\
\hline HAS - n (\%) & $\begin{array}{c}22 \\
(18,2 \%)\end{array}$ & $\begin{array}{c}21 \\
(24,4 \%)\end{array}$ & $\begin{array}{c}1 \\
(2,9 \%)\end{array}$ & $0,004^{(\mathrm{c})}$ \\
\hline Obesidade $-\mathrm{n}(\%)$ & $\begin{array}{c}16 \\
(13,2 \%)\end{array}$ & $\begin{array}{c}15 \\
(17,4 \%)\end{array}$ & $\begin{array}{c}1 \\
(2,9 \%)\end{array}$ & $0,037^{(c)}$ \\
\hline Sedentarismo - n (\%) & $\begin{array}{c}62 \\
(51,2 \%)\end{array}$ & $\begin{array}{c}45 \\
(52,3 \%)\end{array}$ & $\begin{array}{c}17 \\
(48,6 \%)\end{array}$ & $0,841^{(d)}$ \\
\hline Diabetes mellitus - n (\%) & $\begin{array}{c}10 \\
(8,3 \%)\end{array}$ & $\begin{array}{c}9 \\
(10,5 \%)\end{array}$ & $\begin{array}{c}1 \\
(2,9 \%)\end{array}$ & $0,278^{(c)}$ \\
\hline História familiar - n (\%) & $\begin{array}{c}93 \\
(76,9 \%)\end{array}$ & $\begin{array}{c}68 \\
(79,1 \%) \\
\end{array}$ & $\begin{array}{c}25 \\
(71,4 \%) \\
\end{array}$ & $0,476^{(d)}$ \\
\hline
\end{tabular}

Distribuição dos fatores de risco para doenças cardiovasculares em indivíduos moradores do Bairro Barrinha, Viçosa/MG. $\mathrm{n}$ = tamanho da amostra, IMC = índice de massa corporal, HAS = hipertensão arterial sistêmica, $\mathrm{p}=$ probabilidade para os testes de hipóteses. $\left({ }^{\mathrm{a}}\right)$ teste Mann-Whitney, dados apresentados como mediana e diferença interquartil; $\left({ }^{b}\right)$ teste t-Student, dados apresentados como média e desvio padrão; $\left({ }^{c}\right)$ teste exato de

Fisher e $\left.{ }^{d}\right)$ teste de Qui-quadrado, dados apresentados como número de participantes e porcentagem.

lência dos fatores de risco obtida para os participantes do estudo. A prevalência de tabagismo, obesidade e HAS foi significativamente maior em adultos quando comparados com crianças e adolescentes (Tabela 1).

Entre os fatores de risco analisados os mais prevalentes foram história familiar de doenças cardiovasculares e sedentarismo, presentes em $76,9 \%$ e $51,2 \%$ dos indivíduos estudados ( $\mathrm{n}=121)$, respectivamente.

Quando os participantes foram divididos por idade e sexo, não foram observadas diferenças significativas entre os grupos, dados apresentados nas Tabelas 2 e 3.

Foram observadas correlações positivas e significativas entre as variáveis idade, IMC, circunferência da cintura, pressão arterial sistólica e diastólica quando comparadas de forma univariada. Na Tabela 4 estão detalhadas tais correlações com seus respectivos índices e probabilidades. Entre as demais variáveis não foram observadas correlações estatisticamente significativas. 
Tabela 2. Fatores de risco cardiovascular em adultos participantes do estudo

\begin{tabular}{|c|c|c|c|c|}
\hline Parâmetro & Total & Homens & Mulheres & $\mathbf{p}$ \\
\hline $\mathrm{n}$ & $86(100 \%)$ & $32(71,1 \%)$ & $54(28,9 \%)$ & - \\
\hline Idade (anos) & $42(27-64)$ & $44,5(33-64)$ & $40(30-63)$ & $<0,848^{(a)}$ \\
\hline $\operatorname{IMC}\left(\mathrm{Kg} / \mathrm{m}^{2}\right)$ & $25,8 \pm 5,3$ & $24,9 \pm 4,6$ & $26,3 \pm 5,7$ & $<0,235^{\text {(b) }}$ \\
\hline Circunferência da cintura (cm) & $89,8 \pm 13,7$ & $89,3 \pm 13,6$ & $90,1 \pm 13,9$ & $<0,771^{\text {(b) }}$ \\
\hline Tabagismo - n (\%) & $15(17,4 \%)$ & $3(9,4 \%)$ & $12(22,2 \%)$ & $0,129^{(\mathbf{c})}$ \\
\hline HAS - n (\%) & $21(24,4 \%)$ & $6(18,8 \%)$ & $15(27,8 \%)$ & $0,346^{(\mathrm{d})}$ \\
\hline Obesidade - n (\%) & $15(17,4 \%)$ & $4(12,5 \%)$ & $11(20,4 \%)$ & $0,397(\mathrm{c})$ \\
\hline Sedentarismo - n (\%) & $45(52,3 \%)$ & $19(59,4 \%)$ & $26(48,1 \%)$ & $0,314^{(\mathrm{d})}$ \\
\hline Diabetes mellitus - n (\%) & $9(10,5 \%)$ & $1(3,1 \%)$ & $8(14,8 \%)$ & $0,145^{(\mathrm{c})}$ \\
\hline História familiar - n (\%) & $68(79,1 \%)$ & $22(68,8 \%)$ & $46(85,2 \%)$ & $0,070^{(d)}$ \\
\hline
\end{tabular}

Distribuição dos fatores de risco para doenças cardiovasculares em indivíduos adultos moradores do Bairro Barrinha, Viçosa/MG, de acordo com o sexo. $\mathrm{n}$ = tamanho da amostra, IMC = índice de massa corporal, HAS = hipertensão arterial sistêmica, $\mathrm{p}=$ probabilidade para os testes de hipóteses. $\left(^{\mathrm{a}}\right)$ teste Mann-Whitney, dados apresentados como mediana e diferença interquartil; $\left({ }^{b}\right)$ teste t-Student, dados apresentados como média e desvio padrão; $\left({ }^{c}\right)$ teste exato de Fisher e ${ }^{(\mathrm{d})}$ teste de Qui-quadrado, dados apresentados como número de participantes e porcentagem.

Tabela 3. Fatores de risco cardiovascular em crianças e adolescentes participantes do estudo

\begin{tabular}{|c|c|c|c|c|}
\hline Parâmetro & Total & Sexo masculino & Sexo Feminino & $\mathbf{p}$ \\
\hline $\mathrm{n}$ & $35(100 \%)$ & $14(40 \%)$ & $21(60 \%)$ & - \\
\hline Idade (anos) & $10(7-12)$ & $7(6-12)$ & $10(9-11,8)$ & $<0,154^{(a)}$ \\
\hline $\operatorname{IMC}\left(\mathrm{Kg} / \mathrm{m}^{2}\right)$ & $16,9(15,2-18,9)$ & $17,4(15,8-20,1)$ & $16,5(14,6-18,1)$ & $<0,183^{\text {(b) }}$ \\
\hline Circunferência da cintura $(\mathrm{cm})$ & $62,2 \pm 12,5$ & $59(53,8-71,3)$ & $63,5(58,5-65,2)$ & $<0,610^{\text {(b) }}$ \\
\hline Tabagismo - n (\%) & $1(2,9 \%)$ & - & $1(4,8 \%)$ & - \\
\hline HAS - n (\%) & $1(2,9 \%)$ & - & $1(4,8 \%)$ & - \\
\hline Obesidade - n (\%) & $1(2,9 \%)$ & $1(7,1 \%)$ & - & - \\
\hline Sedentarismo - n (\%) & $17(48,6 \%)$ & $6(42,9 \%)$ & $11(52,4 \%)$ & $0,581^{(\mathrm{c})}$ \\
\hline Diabetes mellitus - n (\%) & $1(2,9 \%)$ & $1(7,1 \%)$ & - & - \\
\hline História familiar - n (\%) & $25(71,4 \%)$ & $8(57,1 \%)$ & $17(80,9 \%)$ & $0,151^{(c)}$ \\
\hline
\end{tabular}

Distribuição dos fatores de risco para doenças cardiovasculares em crianças e adolescentes do Bairro Barrinha, Viçosa/

$\mathrm{MG}$, de acordo com o sexo. $\mathrm{n}=$ tamanho da amostra, $\mathrm{IMC}=$ índice de massa corporal, HAS = hipertensão arterial sistêmica, $\mathrm{p}=$ probabilidade para os testes de hipóteses. $\left({ }^{\mathrm{a}}\right)$ teste Mann-Whitney, dados apresentados como mediana e diferença interquartil; $\left({ }^{b}\right)$ teste t-Student, dados apresentados como média e desvio padrão; ( $\left.{ }^{c}\right)$ teste de Qui-quadrado, dados apresentados como número de participantes e porcentagem. 
Tabela 4. Correlações significativas observadas entre as variáveis estudadas $(\mathrm{p}<0,001)$.

\begin{tabular}{ccc}
\hline Variáveis & $\mathbf{r}$ & $\mathbf{p}$ \\
\hline Idade X Circunferência da cintura & 0,71 & $<0,001$ \\
Idade X IMC & 0,62 & $<0,001$ \\
Idade X Pressão arterial sistólica & 0,65 & $<0,001$ \\
Idade X Pressão arterial diastólica & 0,52 & $<0,001$ \\
IMC X Circunferência da cintura & 0,58 & $<0,001$ \\
IMC X Pressão arterial sistólica & 0,34 & $<0,001$ \\
IMC X Pressão arterial diastólica & 0,36 & $<0,001$ \\
Circunferência da cintura X Pressão \\
arterial sistólica
\end{tabular}

$\mathrm{r}=$ coeficiente de correlação de Pearson, $\mathrm{IMC}=$ índice de massa corporal, $\mathrm{p}=$ probabilidades para os testes de correlação.

\section{Discussão}

A análise dos dados do estudo permitiu fazer um desenho dos principais FRC da região estudada. O estudo pontuou os FRC modificáveis e não-modificáveis e foi possível a construção de um perfil da comunidade de modo a proporcionar, futuramente, melhor investimento nas estratégias de abordagem dessa população no âmbito dos cuidados em saúde. O presente estudo firma-se como importante ferramenta para o entendimento da prevalência dos determinados FRC na população em questão. A necessidade de identificação de tais fatores baseia-se no amplo espectro de terapias preventivas existentes hoje, sendo possível organizar estratégias específicas para os diferentes perfis de risco, levando em consideração a disponibilidade de intervenções1.

Ao avaliarmos os FRC modificáveis e não modificáveis entre os adultos não houve diferença estatística na prevalência dos mesmos entre homens e mulheres (Tabela 2). Nesse aspecto a amostra mostrou-se homogênea quanto à distribuição desses FRC. Entre as crianças e adolescentes também não houve diferença entre os gêneros (Tabela 3). Ao analisarmos a prevalência dos FRC nos dois grupos, adultos e crianças, percebe-se diferença significativamente maior da presença dos fatores tabagismo, obesidade e HAS nos adultos (Tabela 1).

Destacam-se como FRC mais prevalentes (Figura 1) em participantes do estudo o sedentarismo $(51,2 \%)$ e a história familiar para DCV $(76,9 \%)$, havendo predomínio do último quando avaliados os grupos separadamente e por sexo. A história familiar representa a predisposição genética de cada indivíduo e como supracitado, é con- siderada um fator de risco não modificável, Destaca-se, entretanto, que o sedentarismo enquadra-se como fator de risco modificável e pode tornar-se alvo de ações preventivas na comunidade. Um estudo realizado na cidade de Bauru-SP, por Monteiro e colaboradores (2007) introduziu durante quatro meses atividades físicas regulares entre hipertensas usuárias de uma Unidade Básica de Saúde, sendo observado ao final melhora da função cardiovascular ${ }^{7}$, inclusive no controle dos níveis pressóricos dentro da normalidade. Ao longo da vida soma-se ao sedentarismo o aparecimento de doenças crônicas que, frequentemente reduzem o nível de atividade física que, por sua vez expõem o indivíduo ao maior risco de doenças e à incapacidade funcional ${ }^{8}$.

$\mathrm{Na}$ análise comparativa entre as faixas etárias houve significativa diferença percentual entre os fatores tabagismo, HAS, obesidade e Diabetes mellitus, os quais foram identificados três a oito vezes mais elevados nos adultos em comparação ao grupo das crianças e adolescentes (Tabela 1). Embora as manifestações clínicas das DCV sejam frequentemente observadas na fase adulta, a presença de fatores de risco na infância ou adolescência é um forte preditor de DCV na idade adulta, por isso a importância da identificação e comparação dos FRC nas diversas faixas etárias ${ }^{9}$. Apesar de a tabela 2 não ter demonstrado diferenças significativas para os FRC entre homens e mulheres, torna-se necessário enfatizar que o tabagismo, a HAS, a obesidade e o Diabetes mellitus mostraram-se mais prevalentes nas mulheres, o que pode demonstrar uma menor aderência do sexo feminino a atividades e atitudes de proteção cardiovascular, como a prática de exercícios físicos e o consumo diário de ve- 
getais e frutas ${ }^{10}$. Apenas o sedentarismo, também fator de risco modificável, foi mais prevalente nos homens $(59,4 \%)$. Em estudo prévio, cujo público analisado foi os servidores da Universidade Federal de Viçosa, avaliando o IMC e sua correlação com FRC cardiovascular, no ano de 2006, também não foram observadas diferença significativas entre os sexos quando avaliada a PAS e PAD11. Entretanto, maior frequência de tabagismo foi observada no sexo masculino. Outro estudo realizado também na Universidade Federal de Viçosa, tendo como amostra os estudantes de graduação, apontou diferenças significativas entre o IMC e sedentarismo, superior nas mulheres em relação aos homens ${ }^{12}$.

A correlação entre as medidas de IMC obtidas e classificação do IMC adaptada pela OMS, revela que os homens da população estudada apresentam-se com sobrepeso $\left(24,9 \mathrm{Kg} / \mathrm{m}^{2}\right)$ e as mulheres enquadram-se em pré-obesas $\left(26,3 \mathrm{Kg} / \mathrm{m}^{2}\right)$. Destaca-se ainda que a média do IMC de adultos participantes do estudo foi de $25,7 \mathrm{Kg} /$ $\mathrm{m}^{2}$, o que os classifica em pré-obesos $(25-29,9)$ segundo a OMS, valor que aumenta o risco de comorbidades ${ }^{13}$.

Em análise da população de crianças e adolescentes (Tabela 3), não foi encontrada amostra de tabagistas e hipertensos no sexo masculino, enquanto no sexo feminino a taxa foi de 4,8\% para ambos esses fatores, referente a uma adolescente usuária de cigarro. Tal fato é preocupante, tendo em vista que pode trazer consequências em longo prazo, de forma a aumentar a vulnerabilidade a doenças respiratórias, patologias bucais e provocar redução na capacidade física ${ }^{14}$. Enquanto isso, a obesidade e o diabetes mellitus são fatores de risco não presentes no sexo feminino, atingindo 7,1\% do sexo masculino. Dado que todos os FRC citados acima são modificáveis, ações de educação em saúde sobre diminuição dos FRC nessa população jovem será útil para a geração de adultos conscientizados. O sedentarismo e a história familiar, presentes em ambos os sexos, foram mais prevalentes no sexo feminino, com $52,4 \%$ e $80,9 \%$, respectivamente. Assim como em adultos, a história familiar demonstrou ser o FRC mais expressivo para o sexo feminino.

Houve correlação positiva e significativa (Tabela 4) entre idade e circunferência da cintura $(71 \%)$, idade e IMC (62\%), idade e PAS (65\%) e entre idade e PAD (52\%); o que demonstra a considerável interferência da idade nos fatores citados, considerando que a maior idade poderia significar um acúmulo de atitudes e pensamentos desfavoráveis à proteção cardiovascular, resultando em aumento nos parâmetros citados. As correlações positivas observadas entre IMC e PAS (34\%) e entre IMC e PAD (36\%) são, apesar de menores que as anteriores, relevantes, demonstrando a associação entre o IMC e maiores valores nas pressões arteriais. A circunferência da cintura também se correlacionou de forma positiva a maiores valores na PAS (39\%) e PAD (43\%).

\section{Conclusões}

Diante da alta prevalência de FRC modificáveis e não modificáveis observado na população estudada, faz-se necessário o planejamento e concretização de ações de cuidado integral com enfoque na prevenção das DCV junto à comunidade estudada. Especial enfoque deve ser dado à prevenção de FRC modificáveis, já que esses são responsáveis por mais de $90 \%$ do risco atribuível a DCV. Os dados obtidos com o estudo podem ser utilizados para o planejamento, junto à rede municipal de saúde, de programas que incentivem hábitos que contribuam para a diminuição do risco cardiovascular, notadamente abordando os FRC modificáveis e o alerta e informação acerca dos FRC não-modificáveis. A abordagem preventiva poderá ser enfatizada também para crianças e adolescentes, enquanto que nos adultos tornam-se necessárias ações para a diminuição dos FRC já observados, vislumbrando, ao final, a eliminação desses fatores.

\section{Referências}

MINISTÉRIO DA SAÚDE. Secretaria de Atenção à Saúde. Prevenção clínica de doença cardiovascular, cerebrovascular e renal crônica. In: Cadernos de Atenção Básica, (14): Brasília; 2006.

WORLD HEALTH ORGANIZATION. Health topics. Disponível em: http://www.who.int/ cardiovascular_diseases/en/. Acesso em 8 dez 2013.

ORGANIZAÇÃO MUNDIAL DE SAÚDE. Cuidados inovadores para condições crônicas: componentes estruturais de ação. Relatório Mundial. Brasília (DF): OMS; 2003. Disponível em:<http://www. who.int/chp/knowledge/publications/iccc_exec_ summary_port.pdf>

RIBEIRO, Rodrigo A. et al . Custo anual do manejo da cardiopatia isquêmica crônica no Brasil: perspectiva pública e privada. Arq. Bras. Cardiol. São Paulo, v. 85, n. 1, jul. 2005. Disponível em: <http://www. scielo.br/scielo.php?script=sci_arttext\&pid=S0066$782 X 2005001400002 \& \operatorname{lng}=$ pt\&nrm $=$ iso $>$. acessos em 17 fev. 2014. http://dx.doi.org/10.1590/S0066782X2005001400002.

Rosengren, Annika et al. Association of psychosocialrisk factors with risks of acute myocardial infarction in11119 cases and 13648 controls from 52 countries (the INTERHEART study): case-control study. Lancet. V. 364. Set 2004. Disponível em < www.thelancet.com Vol 364 September 11, 2004>. 
Polanczyk, C. A. et al. Fatores de risco cardiovascular no Brasil: os próximos 50 anos! Arq. Bras. Cardiol., São Paulo, v. 84, n. 3, Mar 2006. Disponível em < http://www.scielo.br/pdf/abc/v84n3/a01v84n3.pdf>.

MONTEIRO, Henrique L. et al . Efetividade de um programa de exercícios no condicionamento físico, perfil metabólico e pressão arterial de pacientes hipertensos. Rev. Bras. Med. Esporte, Niterói , v. 13, n. 2, Apr. 2007 . Available from <http://www. scielo.br/scielo.php?script=sci_arttext\&pid=S1517 $86922007000200008 \& \operatorname{lng}=e n \& n r m=i s o>$. Access on 17 Feb. 2014. http://dx.doi.org/10.1590/ S151786922007000200008.

COELHO, Christianne de Faria; BURINI, Roberto Carlos. Atividade física para prevenção e tratamento das doenças crônicas não transmissíveis e da incapacidade funcional. Rev. Nutr., Campinas, v. 22, n. 6, dez. 2009. Disponível em <http://www. scielo.br/scielo.php?script=sci_arttext\&pid=S1415$52732009000600015 \& \operatorname{lng}=\mathrm{pt \& nrm}=\mathrm{iso}>$. Acessos em 17 fev. 2014. http://dx.doi.org/10.1590/ S141552732009000600015.

FARIAS JUNIOR, José Cazuza de et al . Fatores de risco cardiovascular em adolescentes: prevalência e associação com fatores sociodemográficos. Rev. bras. epidemiol., São Paulo , v.14, n.1, Mar.2011. Disponível em <http://www.scielo.br/scielo. php?script=sci_arttext\&pid=S1415-790X20110001 00005\&lng=pt\&nrm=iso $>$. Acessos 17 fev. 2014. http://dx.doi.org/10.1590/S1415-790X2011000100005.

CHEHUEN, Marcel da Rocha et al . Risco cardiovascular e prática de atividade física em crianças e adolescentes de Muzambinho/ MG: influência do gênero e da idade. Rev. Bras. Med. Esporte, São Paulo, v. 17, n. 4, ago. 2011. Disponível em<http://www.scielo. br/scielo.php?script=sci_arttext\&pid=S1517$86922011000400003 \& \operatorname{lng}=$ pt\&nrm=iso $>$. Acessos em 17 fev. 2014. http://dx.doi.org/10.1590/ S151786922011000400003.

REZENDE, Fabiane Aparecida Canaan et al. Índice de massa corporal e circunferência abdominal: associação com fatores de risco cardiovascular. Arq. Bras. Cardiol., São Paulo, v. 87, n. 6, Dec. 2006 - Available from <http://www.scielo.br/scielo. php?script=sci_arttext\&pid=S0066-782X200600190 0008\&lng=en\&nrm=iso $>$. access on 17 Feb. 2014. http://dx.doi.org/10.1590/S0066-782X2006001900008.
Morais, Charles Augusto dos Santos et al. Fatores de risco cardiovascular em estudantes de graduação da Universidade Federal de Viçosa-MG. Rev. Inst. Ciências da Saúde, Minas Gerais, v. 29, n. 4, Out/ Dez. 2011. Disponível em: http://www.unip.br/ comunicacao/publicacoes/ics/edicoes/2011/04_outdez/V29_n4_2011_p261-264.pdf. Ou coloco J Health Sci Inst??

Associação Brasileira para o Estudo da Obesidade e da Síndrome Metabólica Diretrizes brasileiras de obesidade 2009/2010 / ABESO - Associação Brasileira para o Estudo da Obesidade e da Síndrome Metabólica. - 3.ed. - Itapevi, SP : AC Farmacêutica, 2009.

NOGUEIRA, Katia Telles et al. Tabagismo em adolescentes numa escola da rede pública do estado do Rio de Janeiro. Rev. Adolesc. Saúde, Rio de Janeiro, v. , n. 4, out/dez. 2004. Disponível em http://www.adolescenciaesaude.com/detalhe_ artigo.asp?id=199 